\title{
A survey of the clinical management of pediatric patients with asymptomatic central venous catheter-associated venous thromboembolism in Saudi Arabia
}

\author{
Bader Allahyani ${ }^{1}$, Abdullah Ali ${ }^{1}$, Joshua Feder ${ }^{1}$, Abdulrahman Alsultan ${ }^{2}$, Mohammed \\ AlShahrani $^{3}$, and Azzah Alzahrani ${ }^{3}$ \\ ${ }^{1}$ University of Ottawa \\ ${ }^{2}$ King Saud University \\ ${ }^{3}$ Prince Sultan Military Medical City
}

September 11, 2020

\begin{abstract}
Background: Central venous catheters (CVCs) are the main cause of venous thromboembolism (VTE) in critically ill children. The optimal first-line treatment for children with asymptomatic CVC-related VTE is unknown. Due to a paucity of clinical trials, clinical practice guidelines can offer only weak recommendations for the management of asymptomatic CVC- related VTE. Method: This case-based survey was designed to assess current trends in local management strategies for pediatric patients with an asymptomatic CVC- related thrombosis. The survey focused on the use of the thrombophilia testing, management approach, duration of anticoagulation, and the use of secondary prophylaxis. We hypothesize that there will be significant variation in these four management areas, in large part due to the aforementioned paucity of available data.REDCap $\mathbb{R}$ questions were sent to members of the Saudi Arabian Pediatric Hematology/Oncology Society (SAPHOS) clinical forum/email database. We used a hypothetical case scenario to assess management strategies for asymptomatic CVC-related VTE and secondary prophylaxis. Results: Seventy-one (30\%) physicians responded to the survey. The majority of the respondents (83.3\%) did not use thrombophilia testing. The far majority (95\%) treated with anticoagulation. In contrast, the survey respondents varied widely in the duration of anticoagulation and the use of secondary prophylaxis. Conclusions: Asymptomatic CVC-related VTE is a common clinical entity with limited data guiding management. In Saudi Arabia, there remains considerable variability in clinical management. These findings will help identify crucial knowledge gaps in the management of asymptomatic CVC-related VTE and facilitate clinical trials that will help establish evidence-based treatment guidelines
\end{abstract}

\section{Introduction:}

Central venous catheters (CVCs) are the main cause of thrombosis in children, particularly hospitalized, critically ill children ${ }^{1}$. Current evidence suggests an increased incidence in venous thromboembolism (VTE) in the last decade, likely due to increased detection and advanced medical interventions leading to improved survival of previously fatal conditions ${ }^{1,2}$. The incidence of asymptomatic CVC-related VTE varies between studies $(7-35 \%)^{3,4}$. Asymptomatic CVC-related VTE has been associated with other thrombotic risk factors (i.e., cancer, cardiac diseases, and critically ill children $)^{1,5}$. The current literature suggests that the difference in thrombotic burden between asymptomatic and symptomatic VTE in children may influence the differences in complication rates and long-term outcomes (i.e., residual thrombus, recurrence, and post-thrombotic syndrome $(\mathrm{PTS}))^{6}$.

Thrombophilia testing on children with CVC related VTE in the absence of positive family history is not advised by Choosing Wisely $囚$. The current evidence has not shown that thrombophilia testing either predicts recurrence of venous thrombosis or guides the duration of anticoagulant therapy ${ }^{7-10}$ 
Data regarding anticoagulation to prevent recurrent CVC-related VTE in children are scarce. The current guidelines are primarily based on expert opinion regarding the anticoagulation prophylaxis in children with a previously diagnosed CVC-related VTE in whom a new CVC placement is required ${ }^{11}$. Although anticoagulation is not protective against the first episode of CVC-related VTE in children ${ }^{12}$, it seems to have a role in preventing recurrent CVC-related VTE, as secondary prophylaxis ${ }^{13}$.

Although there is reasonable evidence that the use of anticoagulation is safe and effective in children, the implementation of this evidence into daily clinical practice is not straightforward ${ }^{14}$. The previous 2012 Chest guideline from The American College of Chest Physicians (ACCP) recommended treating provoked asymptomatic VTE for 12 weeks, even when the provoking risk factor is no longer present ${ }^{11}$. In contrast, the 2018 guideline from the American Hematology Society (ASH) for symptomatic CVC-related thrombosis suggests that anticoagulation likely to minimizes complications. More so, the same guideline gives an equivocal recommendation for pediatric patients with asymptomatic deep vein thrombosis (DVT) ${ }^{14}$.

This case-based survey was designed to assess the current local management strategies for pediatric patients with an asymptomatic CVC- related VTE with a focus on the use of thrombophilia testing, the management approach, the duration of anticoagulation, and the use of secondary prophylaxis. We hypothesize that there will be a significant variation in these four management areas, in large part due to a paucity of available data.

\section{Method:}

A case-based survey was developed by the authors targeting the four management areas of interest (e.g., the use of the thrombophilia testing, the approach of treatment (anticoagulation vs. observation), duration of treatment, and the use of secondary prophylaxis). The survey was piloted in the primary author's institution and developed based on respondent feedback. The final survey was posted twice (January 15, 2020, and February 15, 2020) on the Saudi Arabian Pediatric Hematology/Oncology Society (SAPHOS) clinical forum/email database. The survey included three demographic questions: the number of years in practice, patient population, and an annual number of thrombosis patients at the respondent's center. Case scenarios with asymptomatic CVC-related thrombosis were utilized. Case management questions included the use of thrombophilia testing, treatment approach, duration of treatment, and secondary prophylaxis with subsequent CVC placement for a 4-year-old male who is admitted for a septic shock from a lung infection. A CVC is placed, and he develops an asymptomatic CVC-related thrombosis in his right subclavian vein found incidentally while performing an ultrasound (US) to evaluate large neck lymph nodes. This is his first thrombotic event, and there is no family history of thrombosis. A week later, a repeat US demonstrates clot resolution. The same CVC remains in place. Six months later, he is admitted to the intensive care unit with a severe trauma injury from motor vehicle accident requiring placement of a new CVC.

Study data were collected and managed using REDCap electronic data capture tools ${ }^{15,16}$.

\section{Results:}

There was a total of $71(30 \%)$ physician responses of SAPHOS's 236 subscribers at the time of posting, $65 / 71(91.5 \%)$ managed patients with VTE, 60/71 (84.5\%) completed the entire survey. Table 1 provides a summary of the respondent's demographics. The case description and responses to management questions are provided in Table 2 .

Case 1 described a 4-year-old female child with an incidentally found asymptomatic CVC-associated upper extremity VTE in the setting of a critical illness. Only $16.7 \%$ of respondents performed a thrombophilia evaluation. The majority (72\%) would initiate anticoagulation, $3 \%$ only used anticoagulation if the thrombophilia testing was abnormal, $20 \%$ only if the repeat US shows propagation of the clot, and $3.3 \%$ elected not to treat.

There was a significant variation in the duration of anticoagulation: $19 \%$ treated for 6 weeks, $41.4 \%$ for 12 weeks, and $29.3 \%$ treated until the CVC was removed regardless of the duration of therapy. 
The patient in case 1 required a follow-up ultrasound a week later, which demonstrated clot resolution. 35\% would continue anticoagulation at prophylactic dosing for the duration of CVC insertion. 5\% would for continuing anticoagulation at therapeutic dosing, for the duration of the CVC. $24 \%$ used therapeutic dosing for 6 weeks, while $22.4 \%$ did so for 12 weeks. $10.3 \%$ elected to stop anticoagulation treatment. Overall, it seems that clot resolution did not influence the duration of treatment among the respondents.

The patient in case 1 required subsequent CVC placement 6 months later. $20 \%$ of respondents placed her on anticoagulation for secondary prophylaxis, whereas $21.4 \%$ only used secondary prophylaxis if there was a previously identified thrombophilia. $57 \%$ did not start secondary anticoagulation prophylaxis.

\section{Discussion:}

The optimal first-line treatment for children with asymptomatic CVC-related VTE is unknown. We surveyed pediatric hematologists and oncologists in Saudi Arabia using specific cases. The results of the surveys demonstrate a wide variation in the management approaches between physicians. While the majority agreed to initiate anticoagulation therapy for the incidentally found asymptomatic CVC-related VTE, there was significant variation observed in the duration of anticoagulation: around $20 \%$ treated for 6 weeks, $40 \%$ for 12 weeks and $30 \%$ treated until the CVC was removed regardless of the length of therapy. We observed a wide variation as well in the use of secondary prophylaxis when the patient needed a new CVC placed.

A lack of published and definitive evidence may explain this heterogenicity of clinical practice and the variety in the recommendations for antithrombotic therapy in children with asymptomatic VTE. However, the current guidance based on a low level of certainty in the evidence about the benefits of treatment ${ }^{14}$.

Adult data show that asymptomatic VTE may have a favorable outcome without anticoagulation therapy ${ }^{17}$. It is common for pediatricians to hesitate to extrapolate recommendations from adult-literature, given the anatomical and pathophysiological differences in children ${ }^{18-20}$. The treatment decision of asymptomatic CVC-related VTE is affected by the presence of provoked thrombogenic factors and the risk of long-term sequelae that may impact the child's quality of life. Lack of definitive evidence may justify why more than $71 \%$ of physicians treated the asymptomatic CVC-related VTE with anticoagulation, which is in keeping with the previous recommendation from the 2012 Chest guideline ${ }^{11}$.

The majority of surveyed physicians did not send a thrombophilia workup on the initial presentation. Interestingly, 20\% would use thrombophilia testing to guide secondary prophylaxis, despite the Chest 2012 and ASH 2018 guidelines recommending against thrombophilia workup in children with CVC- related thrombosis ${ }^{11,14}$. A prospective Canadian study assessed 245 children who had CVC- related VTE with their first CVC, had a thrombophilia workup, and subsequently had a second CVC placed. The study showed 107 recurrent CVC-related VTE in 84 children, highlighting this is a high-risk group for developing recurrence of CVC-related VTE ${ }^{13}$. The study could not find an association between thrombophilia and recurrence of CVC-related VTE, which again supports the current recommendation against sending thrombophilia workup for CVC- related $\mathrm{VTE}^{13}$.

Variation in treatment duration between the current guidelines and the respondents may be due to a lack of pediatric clinical trials that specifically address treatment duration. The KIDS-DOTT is a randomized controlled trial investigating the safety and efficacy of limited treatment duration (6 weeks vs. 12 weeks) in the setting of a provoked DVT in pediatric patients. This study will provide meaningful answers to the ongoing clinical questions ${ }^{21}$. (ClinicalTrials.gov Identifier: NCT00687882)

The resolution of the clot on the subsequent US follow up did not seem to have a significant influence on the duration of proposed anticoagulation management by respondents. However, $35 \%$ of the physicians would consider prophylactic dosing after the resolution of the clot. Interestingly, all the physicians who elected to treat for the duration of CVC placement preferred to step down to prophylactic dosing for the CVC placement period. A limited number of pediatric studies have reported rates of resolution for asymptomatic VTE, and the impact of the resolution on the long-term outcome is currently unestablished. A recent Australian prospective study assessed the long-term consequences of asymptomatic VTE in 189 children 
with un-tunneled CVCs (CVC in jugular or femoral veins for more than 24 hours) in the pediatric intensive care unit (PICU). One hundred forty-six underwent US screening. 22\% (32/146) had asymptomatic CVCrelated VTE and were followed up two years later with repeat imaging. Thirty-one children had persistent asymptomatic CVC-related thrombus; however, despite no treatment, only one child had mild PTS at 2 years follow $\mathrm{up}^{3}$. Larger studies are needed to understand the clinical significance of these findings.

There was a wide variation in the reported use of anticoagulation prophylaxis for subsequent CVC insertion. With another CVC placement 6 months later, the majority of respondents, $57 \%$, would not initiate anticoagulation prophylaxis. Interestingly, $20 \%$ of the respondents would use thrombophilia testing to guide secondary prophylaxis, despite as previously mentioned, the Chest 2012, ASH 2018 guidelines, and ASHASPHO Choosing Wisely(r) recommending against thrombophilia workup in children with CVC- related thrombosis ${ }^{11,14}$. A previous survey of the members of the American Pediatric Hematology and Oncology (ASPHO) demonstrated that $24.4 \%$ of the respondents used thrombophilia testing to guide their decision for secondary prophylaxis ${ }^{22}$ which is consistent with our study observation.

Three randomized controlled trials (RCTs) and a recent systematic review did not prove the benefit of primary anticoagulation prophylaxis for children with $\mathrm{CVC}^{23-27}$. However, none of the guidelines addressed secondary prophylaxis in a rather common clinical scenario, i.e., subsequent CVC insertion following CVCRT. In the aforementioned prospective Canadian study ${ }^{13}$, they used prophylactic anticoagulation for the subsequent CVC placement. When they compared no anticoagulation vs. anticoagulation (prophylactic dose or treatment dose), the study showed an increase in the risk of VTE recurrence among the nonanticoagulation group ${ }^{13}$. This may support the rationale of prophylactic anticoagulation for secondary CVC placement, with an ongoing clinical question surrounding optimal dosing.

Limitations to this study include that only 60 (25\%) providers responded and completed the forum posting, which may represent a biased, small sample and not fully represent the current management of asymptomatic CVC-related VTE in children in Saudi Arabia. Other limitations of self-reporting with a hypothetical scenario, is that decisions may vary from their actual management. This is the first survey that has explicitly focused on evaluating the practices of hematologists and oncologists on the management of asymptomatic CVC-related VTE in children in Saudi Arabia.

Our study has demonstrated that there is considerable variability in management, particularly in the duration of treatment and the use of secondary prophylaxis for pediatric patients with an asymptomatic CVC-related VTE. Further clinical trials are needed to provide evidence-based treatment guidelines.

\section{Declaration}

The authors declare no conflict of interest

\section{Acknowledgments}

The authors would like to thank Dr. Char Witmer (The Children's Hospital of Philadelphia) for providing guidance and feedback on designing the survey and reviewing manuscript, Dr. Robert Klaassen (Children's Hospital of Eastern Ontario) for his guidance as a research lead in the primary author's institution, Dr. Donna Johnston (Children's Hospital of Eastern Ontario) and Dr. Yaser Mohammad (Children's Hospital of Eastern Ontario) for their valuable feedback.

The authors would also like to thank members of the Saudi Arabia Pediatric Hematology Oncology Society (SAPHOS) for their support and consideration in taking part in the survey.

\section{References :}

1. Raffini L, Huang YS, Witmer C, Feudtner C. Dramatic increase in venous thromboembolism in children's hospitals in the United States from 2001 to 2007. Pediatrics . 2009. doi:10.1542/peds.2009-0768

2. Witmer C, Raffini L. Treatment of venous thromboembolism in pediatric patients. Blood . 2020;135(5):335-343. doi:10.1182/blood.2019001847 
3. Jones S, Butt W, Monagle P, Cain T, Newall F. The natural history of asymptomatic central venous catheter-related thrombosis in critically ill children. Blood . 2019. doi:10.1182/blood-2018-05-849737

4. Talbott GA, Winters WD, Bratton SL, O'rourke PP. A Prospective Study of Femoral Catheter-Related Thrombosis in Children. Arch Pediatr Adolesc Med . 1995. doi:10.1001/archpedi.1995.02170150068012

5. Mitchell LG, Andrew M, Abshire T, et al. A prospective cohort study determining the prevalence of thrombotic events in children with acute lymphoblastic leukemia and a central venous line who are treated with L-asparaginase: Results of the Prophylactic Antithrombin Replacement in Kids with Acute. Cancer . 2003. doi: $10.1002 /$ cncr. 11042

6. Monagle P. Slow progress. How do we shift the paradigm of thinking in pediatric thrombosis and anticoagulation? Thromb Res . 2019. doi:10.1016/j.thromres.2018.07.016

7. Baglin T, Gray E, Greaves M, et al. Clinical guidelines for testing for heritable thrombophilia. $\mathrm{Br} J$ Haematol . 2010. doi:10.1111/j.1365-2141.2009.08022.x

8. Chalmers E, Ganesen V, Liesner R, et al. Guideline on the investigation, management and prevention of venous thrombosis in children. Br J Haematol . 2011. doi:10.1111/j.1365-2141.2010.08543.x

9. National Institute for Health and Care Excellence. Venous thromboembolic diseases : diagnosis , management and thrombophilia testing. NICE Guidel . 2015.

10. Choosing Wisely. American Society of Hematology-American Society of Pediatric Hematology/Oncology. Don't order thrombophilia testing on children with venous access (i.e., peripheral or central) associated thrombosis in the absence of a positive family history. Released December 9, 2019. https://www.choosingwisely.org/clinician-lists/ash-aspho-avoid-thrombophilia-testing-in-childrenwithout-positive-family-history/. Accessed July 8, 2020

11. Monagle P, Chan AKC, Goldenberg NA, et al. Antithrombotic therapy in neonates and children: Antithrombotic therapy and prevention of thrombosis, 9th ed: American college of chest physicians evidencebased clinical practice guidelines. Chest . 2012. doi:10.1378/chest.11-2308

12. Brandao LR, Shah N, Shah PS. Low molecular weight heparin for prevention of central venous catheterization-related thrombosis in children. Cochrane Database Syst Rev . 2014. doi:10.1002/14651858.CD005982.pub2

13. Avila ML, Amiri N, Stanojevic S, et al. Can thrombophilia predict recurrent catheter-related deep vein thrombosis in children? Blood . 2018. doi:10.1182/blood-2017-10-811216

14. Monagle P, Cuello CA, Augustine C, et al. American Society of Hematology 2018 Guidelines for management of venous thromboembolism: Treatment of pediatric venous thromboembolism. Blood Adv . 2018. doi:10.1182/bloodadvances.2018024786

15. Harris PA, Taylor R, Thielke R, Payne J, Gonzalez N, Conde JG. A metadata-driven methodology and workflow process for providing translational research informatics support, J Biomed Inform. J Biomed Inf . 2009. doi:10.1016/j.jbi.2008.08.010.Research

16. Harris PA, Taylor R, Minor BL, et al. The REDCap consortium: Building an international community of software platform partners.J Biomed Inform . 2019. doi:10.1016/j.jbi.2019.103208

17. Echigoya R, Okamoto H, Uchino H, et al. Incidence and outcome of asymptomatic deep vein thrombosis in critically ill patients: a prospective cohort study. Crit Care . 2015. doi:10.1186/cc14406

18. Andrew M, Paes B, Milner R, et al. Development of the human coagulation system in the full-term infant. Blood . 1987. doi:10.1182/blood.v70.1.165.bloodjournal701165

19. Andrew M, Paes B, Johnston M. Development of the hemostatic system in the neonate and young infant. J Pediatr Hematol Oncol . 1990. doi:10.1097/00043426-199021000-00019 
20. Andrew M, Paes B, Milner R, et al. Development of the human coagulation system in the healthy premature infant. Blood . 1988. doi:10.1182/blood.v72.5.1651.bloodjournal7251651

21. Goldenberg NA, Abshire T, Blatchford PJ, et al. Multicenter randomized controlled trial on Duration of Therapy for Thrombosis in Children and Young Adults (the Kids-DOTT trial): Pilot/feasibility phase findings. J Thromb Haemost . 2015. doi:10.1111/jth.13038

22. Witmer CM, Sauck E, Raffini LJ. A survey of pediatric hematology/oncology specialists regarding management of central line associated venous thrombosis. Pediatr Blood Cancer . 2017;64(7):7-10. doi: $10.1002 /$ pbc. 26383

23. Massicotte P, Julian JA, Gent M, et al. An open-label randomized controlled trial of low molecular weight heparin for the prevention of central venous line-related thrombotic complications in children: The PROTEKT trial. Thromb Res . 2003. doi:10.1016/S0049-3848(03)00099-9

24. Ruud E, Holmstrom H, De Lange C, Hogstad EM, Wesenberg F. Low-dose warfarin for the prevention of central line-associated thromboses in children with malignancies - A randomized, controlled study. Acta Paediatr Int J Paediatr . 2006. doi:10.1080/08035250600729092

25. Schroeder AR, Axelrod DM, Silverman NH, Rubesova E, Merkel E, Roth SJ. A continuous heparin infusion does not prevent catheter-related thrombosis in infants after cardiac surgery*. 2010;11(4):489-495. doi:10.1097/PCC.0b013e3181ce6e29

26. Vidal E, Sharathkumar A, Glover J, Faustino EVS. Central venous catheter-related thrombosis and thromboprophylaxis in children: A systematic review and meta-analysis. J Thromb Haemost . 2014. doi:10.1111/jth.12598

27. Pelland-Marcotte MC, Amiri N, Avila ML, Brandao LR. Low molecular weight heparin for prevention of central venous catheter-related thrombosis in children. Cochrane Database Syst Rev . 2020. doi:10.1002/14651858.CD005982.pub3

\section{Hosted file}

Table 1.docx available at https://authorea.com/users/331658/articles/480447-a-survey-ofthe-clinical-management-of-pediatric-patients-with-asymptomatic-central-venous-catheterassociated-venous-thromboembolism-in-saudi-arabia

\section{Hosted file}

Table 2.docx available at https://authorea.com/users/331658/articles/480447-a-survey-ofthe-clinical-management-of-pediatric-patients-with-asymptomatic-central-venous-catheterassociated-venous-thromboembolism-in-saudi-arabia 